\title{
Gene knockout by viral delivery
}

\author{
Gerald M. Edelman
}

The development of transgenic technology over the past 15 years has facilitated the analysis of the in vivo role of single genes within a genomic network ${ }^{1}$. The application of gene targeting to produce knockout mice is not only a major advance, but the phenotypes that result from the gain or loss of function in transgenic mice have provided insights into the physiological functions of a number of genes during development and have contributed to the understanding of disease.

To analyze those genes that are expressed at multiple times and places during development or in the adult, however, it is necessary to alter gene expression at restricted times or at specific tissue locations. Given the widespread existence of pleiotropy ${ }^{2}$ and of degeneracy $^{3}$, which can lead to compensatory changes following gene deletion, additional means are required for the conditional manipulation of gene expression in a precise spatiotemporal manner. Several recent developments have begun to provide these means.

The report by Rohlmann et $\mathrm{al}^{4}{ }^{4}$ in this issue focuses particular attention on the CreloxP system ${ }^{5}$, which has previously been used to achieve conditional gene activation or deletion (knockout) of targeted mouse genes in specific tissues. These authors combined the use of the Cre-loxP system with an adenovirus delivery technique ${ }^{6,7}$. Recombinant adenovirus can deliver transgenes into specific target tissues. These vectors can efficiently infect a range of cell types, including epithelial cells, endothelial cells, myocytes, and neurons. After injection of the virus into tissues, the high levels of infectivity and the limited spread of the virus results in a localized expression pattern of the transgene in various somatic tissues, including the brain, liver, lung, heart, and muscle. Only a single transgenic line with $l o x P$ sites in a particular location is required to study different tissues at multiple time points. The need to breed Cre recombinase mice with loxP mice ${ }^{5}$ is obviated.

Anton and Graham ${ }^{6}$ were the first to show that adenovirus vectors expressing the Cre recombinase can mediate loxP-dependent DNA recombination in tissue-culture cells. The requisite second step to check whether such vectors could achieve tissue specific targeting in vivo was taken in our laboratory? ${ }^{7}$. We employed a recombinant

Gerald M. Edelman is member and chairman of the Department of Neurobiology, The

Scripps Research Institute, 10550 North Torrey

Pines Road, La Jolla, CA 92037. adenoviral vector, Adv/Cre, that contained the Cre recombinase gene under regulation of the thymidine kinase promoter from herpes simplex virus. After intravenous injection of the $\mathrm{Adv} / \mathrm{Cre}$ vector into adult animals, the liver and spleen showed the highest levels of adenoviral infectivity and recombination. Other tissues, such as kidney, lung, and heart, had lower levels of infection and recombination, and only trace levels of recombination were detected in the brain. However, when the Adv/Cre vector was injected directly into specific regions of the adult brain, recombination was detected in the vicinity of the injection site. In addition, injection of this vector into the forebrains of neonatal mice lead to detectable expression of the rearranged lox $P$ locus arising from recombination in the injected regions for at least 8 weeks. These results demonstrate that the Adv/Cre vector expressing a functional Cre protein is capable of mediating loxpdependent recombination in various tissues and that the recombined locus may in some cases be maintained for an extended period.

This approach has now been significantly extended by Herz and colleagues. They generated a mouse strain in which loxP sites were inserted into the gene encoding the LDL receptor related protein (LRP) which is a cell surface receptor for $\alpha 2$ macroglobulin. In contrast to knockout animals with an inactivated LRP that die early in gestation, the lox $P$ animals injected intravenously with recombinant adenovirus containing $\mathrm{Cre}$ under the cytomegalovirus promoter were viable and showed complete recombination, with functional inactivation of the LRP gene in the liver. This inactivation was reflected by a very reduced rate of clearance of injected labeled $\alpha 2$ macroglobulin, compared to wild-type animals. An absence of LRP was also detected by immunoblotting: LRP remained undetectable 28 days after virus administration. This cleanly executed study clearly shows the efficacy of temporally controlled somatic gene disruption in parenchymal liver cells by adenovirusmediated transfer of Cre recombinase. The approach took advantage of the finding that liver parenchymal cells are almost uniformly infected by intravenously administered adenovirus. This methodology can now be extended to a variety of liver proteins important in metabolism, regeneration, and disease.

The adenoviral-based Cre-loxP system possesses certain distinct advantages over conventional transgenic approaches. Adenoviral vectors have high levels of infectivity in a broad range of cell types across many species. When introduced with timed local delivery techniques, these vectors can allow temporally and spatially specific expression of a foreign gene in discrete regions. Although these advantages recommend the use of this methodology, there are certain limitations. Allowance must be made for possible complications arising from the presence of the viral genome and the consequent host immune response. Moreover, as shown in several of our experiments, the possibility of leakage during injections of the virus, or retrograde neuronal transport of viral particles, may compromise the local specificity of central nervous system infections. In addition, not all cell types within a tissue are equally infected with the adenovirus? ${ }^{7}$. For example, in the kidney, numerous tubular epithelial cells in the cortex are infected, but not cells in the medulla. The use of the Adv/Cre in vivo is therefore likely to be restricted to cell types that are accessible and particularly susceptible to adenovirus infection. In this situation, the specificity of adenoviral vectors could be further restricted by incorporating cell type-specific promoters for the transgene.

Gene regulation acts within a complex network of epistatic interactions and pleiotropic effects. The dependence of such interactions on timing and on phenotypic locales reveals that nonlinear interactions are the rule, not the exception. The network targets are often degenerate-nonisomorphic structures can give isofunctional results ${ }^{3}$. It is, therefore, no surprise that specific deletion, even of important genes, often yields viable and apparently normal phenotypes. To thread our way through the maze of gene interactions will require increasingly refined technical manipulations. Targeted DNA recombination in vitro using viruses containing the Cre recombinase gene is a fine example of a combination of important techniques. Refinement of such approaches should allow us to move to a more sophisticated interpretation of the influence of the genotype on the phenotype. Jasin, M., Moynahan, M.E., and Richardson, C. 1996. Proc. Natl. Acad. Sci. USA 93:8804-8808.

2. Gruneberg, H. 1952. The Genetics of the Mouse. Martinus Nijhoff, The Hague, Netherlands.

3. Edelman, G.M. 1993. Cell Adh. Cormm. 1:1-7.

4. Rohlmann, A., Gotthardt, M., Willnow, T.E., Hammer, R.E., and Herz, J. 1996. Nature Biotechnology 14:1562-1565.

5. Gu, H., Marth, J.D., Orban, P.C., Mossmann, H., and Rajewsky, K. 1994. Science 265:103-106.

6. Anton, M., and Graham, F.L. 1995. J. Virol. $69: 4600-4606$.

7. Wang, Y., Krushel, L.A., and Edelman, G.M. 1996 Proc. Natt. Acad. Sci. USA 93:3932-3936. 